the various therapy options. Kidney biopsy is a mandatory for LN trials, as a gold standard for biomarker studies, and for research on pathophysiology. However, can the initial biopsy really tell us anything about the immunopathogenesis of lupus in spleen and lymph nodes or about which drugs to choose or if the patient will respond to an adequate therapy? Will the recent discovery of EXT1/EXT2 deposits in about $1 / 3$ of patients with Class $\mathrm{V}$ LN help to define subgroups of patients with unique phenotypes and prognosis? Isn 't the scientific attention to a biopsy that confirms the diagnosis and grossly defines intensity of treatment a bit irrational?

Too few kidney biopsies? Serum and urine biomarkers are used to guide immunosuppressive treatment of lupus nephritis, although there is compelling evidence that they poorly indicate persistent LN even in patients with a complete clinical response. The same problem in kidney allograft recipients is addressed by protocol biopsies, why is this not so in LN? A repeat biopsy at 12 months may be of utmost value to guide treatment and to predict long-term prognosis, something the first diagnostic biopsy hardly can do. The REBIOLUP trial will address this question. Other important repeat biopsy indications are the 'partial responder biopsy', the 'smoldering lupus nephritis biopsy', the 'chronic kidney disease progression biopsy' and the 'drug withdrawal biopsy'.

Too many kidney biopsies? The least useful re-biopsy maybe when the patient flares as flare biopsies in patients with a previous Class III-V hardly ever affect treatment decisions. When patients with LN 'flare', drug non-adherence or recent reductions in drug doses are the most common cause, rather than a switch of the underlying disease. Systemic lupus erythematosus (SLE) is chronic autoimmune disease with continued disease activity, albeit at different levels in different patients. Therefore, $\mathrm{LN}$ does not require 'induction' and 'maintenance' therapy, but a constant longterm treatment usually with a combination of immunosuppressive drugs. SLE is heterogenous across individuals but not within an individual. Repeat biopsies are needed to detect inflammation when you are not sure about it, not when it is obvious.

Learning Objectives

- Explain the importance of not overestimating the potential of the first diagnostic biopsy as a source of insights into pathophysiology and predictive power beyond what is known

- Describe the value of treatment response as the main predictor for outcome and the potential use of a protocol biopsy at 1 year in this context because serum and urine markers are unreliable

- Discuss and question the value of a 'flare repeat biopsy', because it rarely affects treatment decisions

- Discuss the use of repeat biopsies in non- or partial responders, smoldering lupus nephritis, CKD progression and before drug withdrawal

- Explain the important concept that SLE is a chronic autoimmune disease that requires constant long-term treatment usually with a drug combination (not transient 'induction and maintenance therapy' with single drugs), identical to how we control persistent alloimmunity in kidney transplant recipients
Debate: In with the new, out with old? Should all patients with lupus nephritis receive new generation therapies in addition to established standard of care?

\section{ALL LUPUS NEPHRITIS PATIENTS SHOULD BE INITIALLY MANAGED WITH BOTH ESTABLISHED STANDARD THERAPIES AND THE NEW GENERATION DRUGS}

YK Onno Teng. Leiden University Medical Center (LUMC), the Netherlands

10.1136/lupus-2021-la.12

The year 2020 has been an extraordinary year in many ways but especially for patients with lupus nephritis (LN), as 2020 saw two novel agents (belimumab and voclosporin) finding FDA-approval for the treatment of LN. These agents will both enrich the therapeutic armamentarium, allowing physicians to better treat their $\mathrm{LN}$ patients, as well as broaden treatment strategies that can be employed.

In this pro-con debate, I will highlight the trial data that have led to the FDA approval of belimumab and voclosporin for the treatment of LN. I will also address the unmet needs these new compounds can address in the treatment of LN. Lastly, the potential treatment strategies emerging from the approval of these new agents will be addressed.

Learning Objectives

- Describe the evidence for the latest FDA-approved drugs for LN: Belimumab and voclosporin

- Describe the current challenges physicians face when treating patients with LN

- Describe possible new treatment strategies for patients with LN

\section{ALL LUPUS NEPHRITIS PATIENTS SHOULD BE INITIALLY MANAGED WITH ESTABLISHED STANDARD THERAPIES ALONE}

Dimitrios Boumpas. National and Kapodistrian University of Athens, Greece

\subsection{6/lupus-2021-la.13}

Lupus nephritis (LN) is extremely heterogenous with most patients responding to standard immunosuppressive therapy within 6-12 months. Post hoc analyses suggest that proteinuria at 12 months represents the best single predictor for longterm renal outcome (i.e., risk for end-stage kidney disease (ESKD) or doubling of serum creatine after 10 years). Accordingly, therapy should aim for proteinuria reduction to below 0.7 gm-1/day by 12 months (complete renal response) with patients with nephrotic range proteinuria reaching this milestone for later, by approximately 24 months. Even patients without a complete response at 12-24 months (defined as proteinuria below $1 \mathrm{gm} /$ day and stable creatinine) have an excellent 10-year prognosis.

Based on the above, rushing into using novel therapies from the beginning has the risk of overtreating the vast majority of patients. This is especially true for patients that receive 
cyclophosphamide as initial induction therapy whereby the benefit of adding biologics such as belimumab is not clear. Of course, people argue that the use of belimumab in such cases may allow faster tapering of glucocorticoids and decrease the risk for renal flares with an added benefit for extrarenal lupus. Another argument often being cited is that even in complete renal responses repeat renal biopsy after therapy shows residual histologic activity.

Still most patients will not flare and identification of those at risk for flare can be done better after initial therapy. Moreover, the clinical significance of histologic activity in complete responders is not clear with only a few of these patients eventually showing evidence of clinical flare. Consistently reported risk factors for a higher disease flare rate include younger age at disease onset, no use of antimalarials, persistent generalized disease activity and serological activity (anti-dsDNA, low complement). Accordingly, in stable patients with improving proteinuria I would consider adding novel therapies after the first year if there is residual proteinuria at the $1-2 \mathrm{gm} /$ day range especially in the presence of risk-factors for flares as defined above.

What do we do for patients with refractory or partial responding disease? According to the 2019 EULAR EDTA recommendations $^{1}$ following failure of first line therapy, all remaining first-line therapies including mycophenolate mofetil (MMF)/ mycophenolic acid (MPA) (2-3 g/day), cyclophosphamide (CY) and calcineurin inhibition (CNI) (especially tacrolimus) as monotherapy or 'multitarget' therapy, are recommended. B-cell depleting therapies such as rituximab, although off-label, are also indicated either as monotherapy or as add-on therapy to $\mathrm{MMF}$ / MPA or CY; complete depletion of circulating B-cells predicted clinical remission at 76 weeks. This has recently been supported by a successful trial of obinutuzumab. ${ }^{2}$ Following a response to rituximab, relapses are not uncommon, but occur after a variable length of time. Repeat dose can be considered to prevent or treat a relapse. Finally, belimumab, when added to standardof-care (including MMF or $\mathrm{CY}$ ), may gradually reduce proteinuria and the risk for kidney flares.

Learning Objectives

- Explain the treatment goals in lupus nephritis and their rationale

- Describe initial therapy and define refractory or residual disease

- Discuss the pros and cons of early vs late introduction of novel agents as add-on regimens in standard therapy

\section{REFERENCES}

1. Fanouriakis A, Kostopoulou M, Cheema K, et al. 2019 Update of the Joint European League Against Rheumatism and European Renal Association-European Dialysis and Transplant Association (EULAR/ERA-EDTA) recommendations for the management of lupus nephritis. Ann Rheum Dis. 2020;79(6):713-23.

2. Marinov AD, Wang H, Bastacky SI, et al. The Type II anti-CD20 Antibody Obinutuzumab (GA101) is More Effective than Rituximab at Depleting B Cells and Treating Disease in a Murine Lupus Model. Arthritis Rheumatol. 2020.

\section{Plenary I: Novel strategies for optimizing outcomes in SLE}

\section{PREVENTING DAMAGE AND REDUCING MORTALITY IN LUPUS: HOW ARE WE DOING?}

Murray Urowitz. University of Toronto, Canada
Damage accrual in patients with lupus is due to both the disease itself and the medications used to treat the disease, especially corticosteroids. The disease courses in patients with lupus vary, with most patients running a relapsing remitting course (RR), a smaller number pursue a permanently active (PA) course, and another minority running a monophasic (M) prolonged remission course. ${ }^{1}$ In an inception cohort of 232 patients followed for 10 years we found that $76 \%$ followed a RR course, $10.8 \%$ a PA course and $11.6 \%$ a $\mathrm{M}$ prolonged remission course. Despite disease activity over time being better controlled in the modern era, patients with RR lupus will spend almost half of their course with active disease, resulting in significant damage accrual over time. In an inception cohort of 73 patients followed for 15 years, with a mean duration on corticosteroids of 117 months, there was a progressive increase in damage and at 15 years with $80 \%$ of the damage items recorded being definitely or possibly corticosteroid related. ${ }^{2}$ Furthermore, it has been shown that early damage is a predictor of mortality. In 263 inception patients followed for 10 years, 190 (72\%) had no early damage and $73(28 \%)$ had early damage. In patients with early damage, $25 \%$ died within 10 years as compared to only $7.3 \%$ with no early damage $(\mathrm{p}=0.0002) .{ }^{3}$ Thus, prevention of damage accrual is a key objective in the management of patients with lupus.

We examined whether damage accrual over a 5-year period is reduced with the prior use of antimalarials. Of an inception cohort of 354 patients who had a first ACR/SLICC score of 0,75 developed damage over the first 5 years and these were matched with 150 controls with no damage. Antimalarials were protective for damage accrual in the first 5 years supporting their use at diagnosis. ${ }^{4}$ Finally belimumab, the first biologic approved for the treatment of lupus, has been assessed for the prevention of long term damage accrual. Patients followed long term from the original belimumab trials, compared with propensity score matched patients from the University of Toronto Lupus cohort matching for 17 clinical variables, showed that belimumab reduced organ damage progression, slowed the rate of organ damage progression and reduced the magnitude of year-to-year organ damage. $^{5}$

Learning Objectives

- Describe damage accrual and the nature of the damage

- Describe how damage accrual is associated with mortality

- Demonstrate that hydroxychloroquine protects against damage accrual

- Demonstrate that belimumab protects against damage accrual

\section{REFERENCES}

1. Tselios K, Gladman DD, Touma Z, et al. Disease course patterns in systemic lupus erythematosus. Lupus. 2019;28(1):114-22.

2. Gladman DD, Urowitz MB, Rahman P, Ibañez D, Tam LS. Accrual of organ damage over time in patients with systemic lupus erythematosus. I Rheumatol. 2003;30(9):1955-9.

3. Rahman P, Gladman DD, Urowitz MB, Hallett D, Tam LS. Early damage as measured by the SLICC/ACR damage index is a predictor of mortality in systemic lupus erythematosus. Lupus. 2001;10(2):93-6.

4. Ruiz-Arruza I, Gladman D, Ibanez D, Urowitz M. Antimalarials protect systemic lupus erythematosus patients from damage accrual during the first five years of the disease. Arthritis Rheum. 2012;64:S611.

5. Urowitz MB, Ohsfeldt RL, Wielage $R C$, et al. Organ damage in patients treated with belimumab versus standard of care: a propensity score-matched comparative analysis. Ann Rheum Dis. 2019;78(3):372-9. 